\title{
Larval to Adult Microvascular Anatomy of the Stomach of the South African Clawed Toad, Xenopus laevis Daudin: A Scanning Electron Microscope Study of Vascular Corrosion Casts
}

\author{
A. Lametschwandtner, M. Höll, U. Lametschwandtner, and B. Minnich* \\ University of Salzburg, Department of Organismic Biology, Vascular and Muscle Research Unit, \\ Hellbrunnerstrasse 34, A-4020 Salzburg, Austria (Europe)
}

While the development and remodeling of tissue components of intestine and stomach of amphibians from larval to adult life have been studied by several authors, little attention has been paid to the stomach microvasculature and its behaviour during this period [1 - 3]. The present study demonstrates by means of resin-made vascular corrosion casts and scanning electron microscopy (SEM) the changes the microvascular bed of the stomach of tadpoles of Xenopus laevis Daudin undergoes during metamorphosis when the herbivorous tadpole becomes a carnivorous juvenile.

The cirulatory system of three tadpoles per developmental stage 55 - 64 (for staging see [4]) was cast via the conus arteriosus with Mercox-Cl-2B (Ladd Res. Inc., Burlington, VT), diluted 4+1 $(\mathrm{v}+\mathrm{v})$ with monomeric methylmethacrylic acid (Fluka Chemicals, Basle, $\mathrm{CH}$ ) at a flow rate of $7 \mathrm{ml} / \mathrm{h}$ [4]. In adult animals (2 females, 1 male) resin injection was done manually via a flexible tubing inserted via the ventricle into the conus arteriosus. For more technical details on vascular casting see $[5-6]$.

In at least one tadpole per stage the vasculature of the stomach was sufficiently well cast and specimens could be studied in the SEM.

The microvascular bed of the adult stomach at metamorphic climax (stage 60) is made up of a dense three-dimensional network of predominantly wide immature imposing capillaries (Fig.1). From stage 60 to 62 vascular patterns do not change significantly and vessels still look immature (Fig. 2). A closer view at the serosal aspect of the stomach reveals many sites of intussusceptive microvascular growth (IMG) which takes part in the growth and maturation of the microvascular bed (Fig. 3). In the transverse sectioned cast the angioarchitecture of the stomach wall and the luminal aspect of the subepithelial capillary plexus become evident (Fig. 4). The close-up view shows that there are many small capillary meshes which outline the tubular glands of the stomach (Fig. 5). A clear vessel hierarchy with distinct arteries and veins is not yet fully present.

The vascular bed of the adult stomach is supplied by the gastric arteries which approach the stomach from the medial aspect (Fig. 6). Their branches immediately pierce the serosa and the muscular layer and run within the submucosa and lamina propria. While outwards directed arterioles feed the muscular layer the main vessels terminate in the subepithelial area to feed the capillary bed beneath the stomach epithelium (Fig. 7). The capillary bed consists of a dense network embracing the tubular gastric glands (Figs. 7 and 8). 
References

[1] N. Millard, R. Soc. S. Africa 30 (1945) 217

[2] N. Millard, R. Soc. S. Africa 32 (1949) 55

[3] A. J. Levine et al., Devel. Biol. 254 (2003) 50

[4] P.D. Nieuwkopp and J. Faber, A normal table of Xenopus development (Daudin). North Holland, Amsterdam 1967.

[5] T. Murakami, Arch. Histol. Jap. 32 (1971) 445

[6] B. Minnich, H. Bartel, H. and A. Lametschwandtner, Microvasc. Res. 64 (2002) 425

[7] This work was funded by the Stiftungs- und Förderungsgesellschaft der Paris Lodron Universität Salzburg. The assistance of S. Tholo, A. Zankl and Dr. W.D. Krautgartner is gratefully acknowledged.
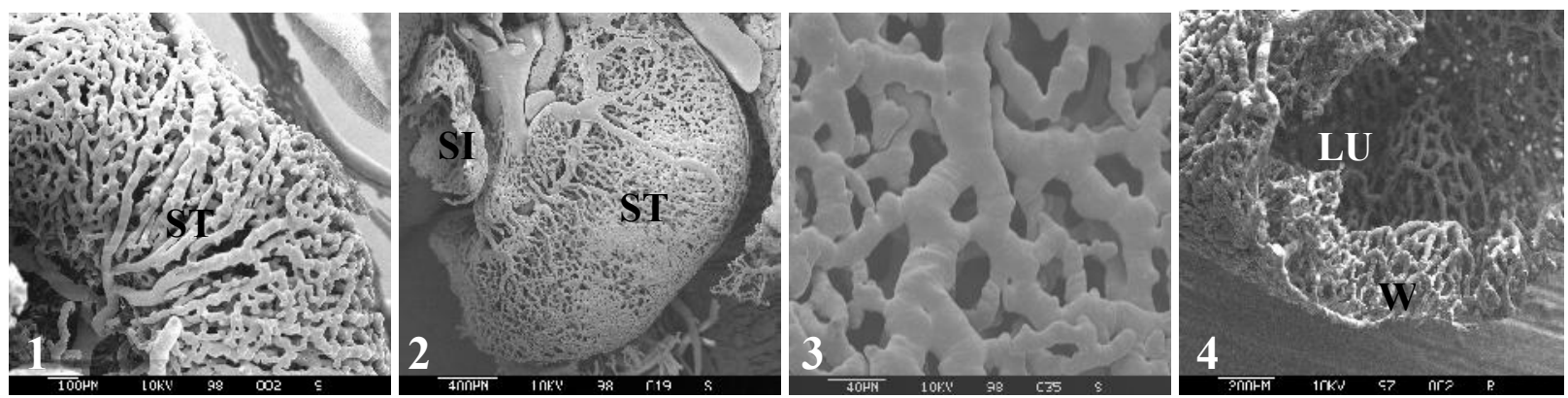

Fig. 1. Stomach of a tadpole of Xenopus laevis at stage 60. Vascular corrosion cast (VCC). ST stomach,. Fig. 2. Stomach at stage 62. SI small intestine. ST stomach. VCC. Fig. 3. Stomach at stage 62. VCC. Serosal aspect. Detail. Note the small holes in the larger vessel indicating sites of intussusceptive microvascular growth (IMG). Fig. 4. Stomach at stage 62/63. VCC. Transverse section. Luminal view. Note the subepithelial vascular pattern.
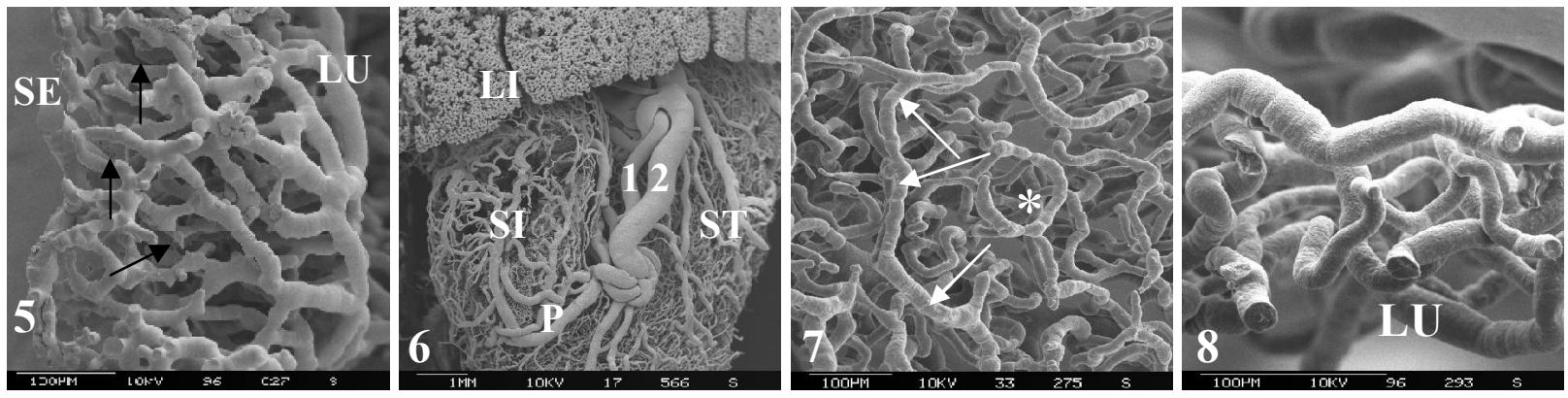

Fig. 5. Microvascular anatomy of the wall of the stomach of a tadpole at stage 63.VCC. LU luminal side, SE serosal side. Arrows indicate the location of the tubular glands. Note the slightly larger vessels at the luminal side. Fig. 6. Stomach of the adult Xenopus.. VCC. Ventral view. LI liver, P pyloric region, SI small intestine, ST stomach, 1 gastric artery, 2 gastric vein. Fig. 7. Subepithelial vascular pattern. Luminal view. Detail . Note the superficial venule (arrows) and the capillary ring (asterisk) indicating the opening of a gastric gland. Fig. 8. Subepithelial capillary bed. Transverse section. LU luminal side. 